\title{
Majorization Results for Certain Subfamilies of Analytic Functions
}

\author{
Muhammad Arif $\mathbb{D D}^{1}{ }^{1}$ Miraj Ul-Haq, ${ }^{1}$ Omar Barukab, ${ }^{2,3}$ Sher Afzal Khan, ${ }^{1}$ \\ and Saleem Abullah ${ }^{1}$ \\ ${ }^{1}$ Faculty of Physical and Numerical Sciences, Abdul Wali Khan University Mardan, Mardan 23200, Pakistan \\ ${ }^{2}$ Faculty of Computing and Information Technology, P.O. Box 344, Rabigh 21911, Saudi Arabia \\ ${ }^{3}$ King Abdulaziz University, Jeddah, Saudi Arabia
}

Correspondence should be addressed to Muhammad Arif; marifmaths@awkum.edu.pk

Received 18 February 2021; Accepted 16 March 2021; Published 8 April 2021

Academic Editor: Gangadharan Murugusundaramoorthy

Copyright (c) 2021 Muhammad Arif et al. This is an open access article distributed under the Creative Commons Attribution License, which permits unrestricted use, distribution, and reproduction in any medium, provided the original work is properly cited.

Let $h_{1}(z)$ and $h_{2}(z)$ be two nonvanishing holomorphic functions in the open unit disc with $h_{1}(0)=h_{2}(0)=1$. For some holomorphic function $q(z)$, we consider the class consisting of normalized holomorphic functions $f$ whose ratios $f(z) / z q(z)$ and $q(z)$ are subordinate to $h_{1}(z)$ and $h_{2}(z)$, respectively. The majorization results are obtained for this class when $h_{1}(z)$ is chosen either $h_{1}(z)=\cos z$ or $h_{1}(z)=1+\sin z$ or $h_{1}(z)=\sqrt{1+z}$ and $h_{2}(z)=1+\sin z$.

\section{Introduction}

In order to better explain the terminology included in our key observations, some of the essential relevant literature on geometric function theory needs to be provided and discussed here. We start with symbol $\mathscr{A}$ which represents the class of holomorphic functions in the region of open unit disc $\mathbb{U}_{d}=$ $\{z \in \mathbb{C}:|z|<1\}$, and if $f(z)$ is in $\mathscr{A}$, then it satisfies the relationship $f(0)=f^{\prime}(0)-1=0$. Also, the family $\mathcal{S} \subset \mathscr{A}$ contains all univalent functions. Though function theory was started in 1851, in 1916, due to coefficient conjecture provided by Bieberbach [1], this field emerged as a good area of new research. This conjecture was proved by De-Branges [2] in 1985. Many good scholars of the period attempted to prove or disprove this conjecture between the years 1916 and 1985. As a result, they identified several subfamilies of a class $\delta$ of univalent functions linked to various image domains. The families of star-like $\mathcal{S}^{*}$ and convex $\mathscr{K}$ functions are the most basic, mostly studied, and beautiful geometric representations of these subfamilies, which are described as

$$
\begin{aligned}
& \mathcal{S}^{*}=:\left\{f \in \mathcal{S}: \mathfrak{R} \frac{z f^{\prime}(z)}{f(z)}>0,\left(z \in \mathbb{U}_{d}\right)\right\}, \\
& \mathscr{K}=:\left\{f \in \mathcal{S}: \mathfrak{R} \frac{\left(z f^{\prime}(z)\right)^{\prime}}{f^{\prime}(z)}>0,\left(z \in \mathbb{U}_{d}\right)\right\} .
\end{aligned}
$$

In 1970, Roberston [3] established the idea of quasisubordination among holomorphic functions. Two functions $\mathscr{F}_{1}(z), \mathscr{F}_{2}(z) \in \mathscr{A}$ are related to the relationship of quasisubordination, denoted mathematically by $\mathscr{F}_{1}(z)<_{q} \mathscr{F}_{2}(z)$, if there exist functions $\varphi(z), u(z) \in \mathscr{A}$ such that $z f^{\prime}(z) / \varphi(z)$ is holomorphic in $\mathbb{U}_{d}$ with $|\varphi(z)| \leqq 1, u(0)=0$, and $|u(z)| \leqq$ $|z|$ satisfying the relationship

$$
\mathscr{F}_{1}(z)=\varphi(z) \mathscr{F}_{2}(u(z)), z \in \mathbb{U}_{d} .
$$

Also, by choosing $u(z)=z$ and $\varphi(z) \equiv 1$, we obtain the most useful concepts of geometric function theory known as subordination between analytic functions. In fact, if 
$\mathscr{F}_{2}(z) \in \mathcal{S}$, then, for $\mathscr{F}_{1}(z), \mathscr{F}_{2}(z) \in \mathscr{A}$, the subordination relationship has

$$
\mathscr{F}_{1}(z) \prec \mathscr{F}_{2}(z) \Leftrightarrow\left[\mathscr{F}_{1}\left(\mathbb{U}_{d}\right) \subset \mathscr{F}_{2}\left(\mathbb{U}_{d}\right) \text { with } \mathscr{F}_{1}(0)=\mathscr{F}_{2}(0)\right] .
$$

By taking $u(z)=z$, the above definition of quasisubordination becomes the majorization between holomorphic functions and is written mathematically by $\mathscr{F}_{1}(z) \ll \mathscr{F}_{2}(z)$, for $\mathscr{F}_{1}(z), \mathscr{F}_{2}(z) \in \mathscr{A}$. That is; $\mathscr{F}_{1}(z) \ll$ $\mathscr{F}_{2}(z)$, if a function $\varphi(z) \in \mathscr{A}$ exists with $|\varphi(z)| \leqq 1$ in such a way that

$$
\mathscr{F}_{1}(z)=\varphi(z) \mathscr{F}_{2}(z), \quad z \in \mathbb{U}_{d}
$$

This idea was introduced by MacGregor [4] in 1967. Numerous articles have been published in which this idea was used. The work of Altintas and Srivastava [5], Cho et al. [6], Goswami and Aouf [7], Goyal and Goswami [8, 9], Li et al. [10], Panigraht and El-Ashwah [11], Prajapat and Aouf [12], and the authors [13, 14] are worth mentioning on this topic.

The general form of the class $\mathcal{S}^{*}$ was studied in 1992 by $\mathrm{Ma}$ and Minda [15] and was given by

$$
\mathcal{S}^{*}(\Lambda)=\left\{f \in \mathcal{S}: \frac{z f^{\prime}(z)}{f(z)} \prec \Lambda(z)\left(z \in \mathbb{U}_{d}\right)\right\}
$$

where $\Lambda(z)$ is a regular function with positive real part and $\Lambda^{\prime}(0)>0$. Also, the function $\Lambda(z)$ maps $\mathbb{U}_{d}$ onto a starshaped region with respect to $\Lambda(0)=1$ and is symmetric about the real axis. They addressed some specific results such as distortion, growth, and covering theorems. In recent years, several subfamilies of the set $\mathscr{A}$ were studied as a special case of the class $\mathcal{S}^{*}(\Lambda)$. For example,

(i) if we take $\Lambda(z)=1+M z / 1+N z$ with $-1 \leq N<M$ $\leq 1$, then the deduced family $\mathcal{S}^{*}[M, N] \equiv \mathcal{S}^{*}(1+M$ $z / 1+N z)$ is described by the functions of the Janowski star-like family established in [16] and later studied in different directions in $[17,18]$

(ii) the family $\mathcal{S}_{L}^{*} \equiv \mathcal{S}^{*}(\Lambda(z))$ with $\Lambda(z)=\sqrt{1+z}$ was developed in [19] by Sokól and Stankiewicz. The image of the function $\Lambda(z)=\sqrt{1+z}$ demonstrates that the image domain is bounded by the Bernoullis lemniscate right-half plan specified by $\left|w^{2}(z)-1\right|$ $<1$

(iii) by selecting $\Lambda(z)=1+\sin z$, the class $\mathcal{S}^{*}(\Lambda(z))$ leads to the family $\mathcal{S}_{\sin }^{*}$ which was explored in [20] while $\mathcal{S}_{e}^{*} \equiv \mathcal{S}^{*}\left(e^{z}\right)$ has been produced in the article [21] and later studied in [22]

(iv) the family $\mathcal{S}_{R}^{*} \equiv \mathcal{S}^{*}(\Lambda(z))$ with $\Lambda(z)=1+z / J(J+$ $z) /(J-z), J=\sqrt{2}+1$ is studied in [23] while $\mathcal{S}_{\cos }^{*}$ $:=\mathcal{S}^{*}(\cos (z))$ and $\mathcal{S}_{\text {cosh }}^{*}:=\mathcal{S}^{*}(\cosh (z))$ were recently examined by Raza and Bano [24], and Abdullah et.al [25], respectively

Now, let us take the nonvanishing analytic functions $h_{1}(z)$ and $h_{2}(z)$ in $\mathbb{U}_{d}$ with $h_{1}(0)=h_{2}(0)=1$. Then, the families defined in this article consist of functions $f(z) \in$ $\mathscr{A}$ whose ratios $f(z) / z q(z)$ and $q(z)$ are subordinated to $h_{1}(z)$ and $h_{2}(z)$, respectively, for some analytic function $q(z)$ with $q(0)=1$ as

$$
\begin{aligned}
\frac{f(z)}{z q(z)} & \prec h_{1}(z), \\
q(z) & \prec h_{2}(z) .
\end{aligned}
$$

We are now going to choose some particular functions instead of $h_{1}(z)$ and $h_{2}(z)$. These choices are

$$
\begin{aligned}
h_{1}(z) & =1+\sin z \\
\text { or } h_{1}(z) & =\cos z \\
\text { or } h_{1}(z) & =\sqrt{1+z}, \\
h_{2}(z) & =1+\sin z,
\end{aligned}
$$

and by applying the above-mentioned concepts, we now consider the following classes:

$$
\mathscr{F}_{\cos }=\left\{f(z) \in \mathscr{A}: \frac{f(z)}{z q(z)} \prec \cos z \& q(z) \prec h_{2}(z), z \in \mathbb{U}_{d}\right\},
$$

$\mathscr{F}_{\delta \mathscr{L}}=\left\{f(z) \in \mathscr{A}: \frac{f(z)}{z q(z)} \prec \sqrt{1+z} \& q(z) \prec h_{2}(z), z \in \mathbb{U}_{d}\right\}$,

$\mathscr{F}_{\text {sin }}=\left\{f(z) \in \mathscr{A}: \frac{f(z)}{z q(z)} \prec 1+\sin z \& q(z) \prec h_{2}(z), z \in \mathbb{U}_{d}\right\}$.

In the present article, we discuss majorization problems for each of the above-defined classes $\mathscr{F}_{\cos }, \mathscr{F}_{\mathcal{S} \mathscr{L}}$, and $\mathscr{F}_{\text {sin }}$.

\section{Main Results}

To prove majorization results for the classes $\mathscr{F}_{\text {cos }}, \mathscr{F}_{\delta \mathscr{L}}$, and $\mathscr{F}_{\text {sin }}$, we need the following lemma.

Lemma 1. Let $q(z) \prec 1+\sin z$ and for $|z| \leq r$. Then, $q(z)$ satisfies the following inequalities:

$$
1-\sin r \cosh r \leq|q(z)| \leq 1+\sin r \cosh r
$$

$$
\left|\frac{z q^{\prime}(z)}{q(z)}\right| \leq \frac{r \cosh r}{\left(1-r^{2}\right)(1-\sinh r)}
$$


Proof. If $q(z) \prec 1+\sin z$, then

$$
q(z)=1+\sin w(z)
$$

for some Schwartz function $w(z)$. Now, after some easy calculations, we have

$$
\frac{z q^{\prime}(z)}{q(z)}=\frac{z w^{\prime}(z) \cos w(z)}{1+\sin w(z)}
$$

Let $w(z)=R e^{i \theta}$ with $|z|=r \leq R,-\pi \leq \theta \leq \pi$.A calculation shows that

$$
\mathfrak{R}\left(\cos \left(R e^{i \theta}\right)\right)=\cos (R x) \cosh (R y),
$$

where

$$
\begin{gathered}
x=\cos \theta, \\
y=\sin \theta, \\
\text { for } x, y \in[-1,1] .
\end{gathered}
$$

Now, we can write

$$
\begin{aligned}
\cos (R x) & \geq \cos R \geq \cos r, \\
1 & \leq \cosh (R y) \leq \cosh R \leq \cosh r .
\end{aligned}
$$

Thus, we have

$$
\Re \cos w(z) \geq \cos r
$$

Now, consider

$$
\begin{aligned}
\left|\sin \left(R e^{i \theta}\right)\right|^{2}= & \cos (R \cos \theta)^{2} \sinh (R \sin \theta)^{2} \\
& +\sin (R \cos \theta)^{2} \cosh (R \sin \theta)^{2} \\
= & \Psi(\theta) .
\end{aligned}
$$

A calculation shows that the numbers, $0, \pm \pi, \pm \pi / 2$, are the roots of equation (19) in $[-\pi, \pi]$. Since $\Psi(\theta)$ is an even function, it is sufficient to consider $\theta \in[0, \pi]$. We observe that $\Psi(\pi / 2)=\sinh ^{2}(R)$ and $\Psi(0)=\sin ^{2}(R)$. Now, we can write

$$
\max \left\{\Psi(0), \Psi\left(\frac{\pi}{2}\right), \Psi(\pi)\right\}=\sin h^{2}(R)
$$

Hence,

$$
\left|\sin R\left(e^{i \theta}\right)\right| \leq \sinh (R) \leq \sinh r
$$

Similarly, one can easily show that

$$
\cos r \leq|\cos w(z)| \leq \cosh r .
$$

Now, from well-known inequality for Schwartz function $w(z)$, we obtain

$$
\left|w^{\prime}(z)\right| \leq \frac{1-|w(z)|^{2}}{1-|z|^{2}}=\frac{1-R^{2}}{1-|z|^{2}} \leq \frac{1}{1-r^{2}} .
$$

Now, by applying (21), (22), and (23) in (14), we get (12).

Theorem 2. Let the functions $f(z) \in \mathscr{A}, g(z) \in \mathscr{F}_{\cos }$ and also suppose that $f(z) \ll g(z)$ in $\mathbb{U}_{d}$. Then, for $|z| \leq r_{1}$,

$$
\left|f^{\prime}(z)\right| \leqq\left|g^{\prime}(z)\right|
$$

where $r_{1}$ is the smallest positive root of the equation

$$
\begin{gathered}
\left(\left(\left(1-r^{2}\right)-r(1+\rho)\right) \cos r-r \sinh r\right) \\
\cdot(1-\sinh r)-r \cos r \cosh r=0
\end{gathered}
$$

Proof. If $g(z) \in \mathscr{F}_{\text {cos }}$, then by the subordination relationship, we have

$$
\frac{g(z)}{z q(z)}=\cos w(z)
$$

Now, after simple calculations, we have

$$
\frac{z g^{\prime}(z)}{g(z)}=1+\frac{z q^{\prime}(z)}{q(z)}-\frac{z w^{\prime}(z) \sin w(z)}{\cos w(z)} .
$$

Now, by using (21), (22), and (23) along with Lemma 1, we obtain

$$
\begin{aligned}
& \left|\frac{g(z)}{g^{\prime}(z)}\right|=\frac{|z|}{\left|1+z q^{\prime}(z) / q(z)-z w^{\prime}(z) \sin w(z) / \cos w(z)\right|} \\
& \quad \leqq \frac{|z|}{1-\left|z q^{\prime}(z) / q(z)\right|-\left|z w^{\prime}(z) \sin w(z) / \cos w(z)\right|} \\
& \quad \leqq \frac{r\left(1-r^{2}\right)(1-\sinh r) \cos r}{\left(1-r^{2}\right)(1-\sinh r) \cos r-r \cos r \cosh r-r \sinh r(1-\sinh r)}
\end{aligned}
$$

From (4), we can write

$$
f(z)=\varphi(z) g(z)
$$

Differentiating the above equality on both sides, we get

$$
\begin{aligned}
f^{\prime}(z) & =\varphi^{\prime}(z) g(z)+\varphi(z) g^{\prime}(z) \\
& =g^{\prime}(z)\left(\varphi(z)+\varphi^{\prime}(z) \frac{z g^{\prime}(z)}{g(z)}\right)
\end{aligned}
$$

Also, the Schwartz function $\varphi(z)$ fulfils the below inequality:

$$
\left|\varphi^{\prime}(z)\right| \leqq \frac{1-|\varphi(z)|^{2}}{1-|z|^{2}}=\frac{1-|\varphi(z)|^{2}}{1-r^{2}}\left(z \in \mathbb{U}_{d}\right) .
$$


Now, applying (28) and (31) in (30), we have

$\left|f^{\prime}(z)\right| \leqq\left[\varphi(z)+\frac{r\left(1-|\varphi(z)|^{2}\right)(1-\sinh r) \cos r}{\left(1-r^{2}\right)(1-\sinh r) \cos r-r \cos r \cosh r-r \sinh r(1-\sinh r)}\right]\left|g^{\prime}(z)\right|$,

which by putting

$$
\left|\varphi^{\prime}(z)\right|=\rho(0 \leqq \rho \leqq 1)
$$

becomes the inequality

$$
\left|f^{\prime}(z)\right| \leqq \Phi_{1}(r, \rho)\left|g^{\prime}(z)\right|
$$

where

$$
\Phi_{1}(r, \rho)=\varphi(z)+\frac{r\left(1-|\varphi(z)|^{2}\right)(1-\sinh r) \cos r}{\left(\left(1-r^{2}\right) \cos r-r \sinh r\right)(1-\sinh r)-r \cos r \cosh r} .
$$

To determine $r_{1}$, it is sufficient to choose

$$
r_{1}=\max \left(r \in[0,1): \Phi_{1}(r, \rho) \leqq 1, \forall \rho \in[0,1]\right),
$$

or, equivalently,

$$
r_{1}=\max \left(r \in[0,1): \Psi_{1}(r, \rho) \geq 0, \forall \rho \in[0,1]\right),
$$

where

$$
\begin{aligned}
\Psi_{1}(r, \rho)= & \left(\left(1-r^{2}-r(1+\rho)\right) \cos r-r \sinh r\right) \\
& \cdot(1-\sinh r)-\cos r \cosh r
\end{aligned}
$$

Clearly, when $\rho=1$, the function $\Psi_{1}(r, \rho)$ assumes its minimum value, namely,

$$
\min \left(\Psi_{1}(r, \rho), \rho \in[0,1]\right)=\Psi_{1}(r, 1)=\Psi_{1}(r),
$$

where

$$
\begin{aligned}
\Psi_{1}(r)= & \left(\left(1-r^{2}-2 r\right) \cos r-r \sinh r\right) \\
& \cdot(1-\sinh r)-r \cos r \cosh r .
\end{aligned}
$$

Next, we have the following inequalities:

$$
\begin{aligned}
& \Psi_{1}(0)=1>0, \\
& \Psi_{1}(1)=-0.43851<0 .
\end{aligned}
$$

There exists $r_{1}$ such that $\Psi_{1}(r) \geq 0$ for all $r \in\left[0, r_{1}\right]$, where $r_{1}$ is the smallest positive root of equation (25). Thus, the proof is completed.

Theorem 3. Let $f(z) \in \mathscr{A}, g(z) \in \mathscr{F}_{\text {sin }}$ and also suppose that $f(z) \ll g(z)$ in $\mathbb{U}_{d}$. Then, for $|z| \leq r_{2}$,

$$
\left|f^{\prime}(z)\right| \leqq\left|g^{\prime}(z)\right|
$$

where $r_{2}$ is the root (smallest positive) of the equation:

$$
\left(1-r^{2}\right)(1-\sinh r)-2 r(\cosh r+1-\sinh r)=0
$$

Proof. If $g(z) \in \mathscr{F}_{\sin }$, then by using (10) along with the subordination relationship, a holomorphic function $w(z)$ in $\mathbb{U}_{d}$ occurs with $w(0)=0$ and $|w(z)| \leq|z|$ in such a way that

$$
\frac{g(z)}{z q(z)}=1+\sin w(z)
$$

hold. Now, after simple calculations, we have

$$
\frac{z g^{\prime}(z)}{g(z)}=1+\frac{z q^{\prime}(z)}{q(z)}+\frac{z w^{\prime}(z) \cos w(z)}{1+\sin w(z)}
$$

Using (21), (22), and (23) along with Lemma 1, we obtain

$$
\begin{aligned}
\left|\frac{g(z)}{g^{\prime}(z)}\right| & =\frac{|z|}{\left|1+z p^{\prime}(z) / p(z)+z w^{\prime}(z) \cos w(z) / 1+\sin w(z)\right|} \\
& \leqq \frac{r\left(1-r^{2}\right)(1-\sinh r)}{\left(1-r^{2}\right)(1-\sinh r)-2 r \cosh r} .
\end{aligned}
$$

Also, with the use of (31) and (46) in (30), we easily get

$$
\left|f^{\prime}(z)\right| \leqq\left[\varphi(z)+\frac{r\left(1-|\varphi(z)|^{2}\right)(1-\sinh r)}{\left(1-r^{2}\right)(1-\sinh r)-2 r \cosh r}\right]\left|g^{\prime}(z)\right| \text {. }
$$

Now, by the similar lines of Theorem 2 along with the virtue of (33), we easily obtain the required result.

Theorem 4. Let $f(z) \in \mathscr{A}, g(z) \in \mathscr{F}_{\mathcal{S} \mathscr{L}}$ and also suppose that $f(z)$ is majorized by $g(z)$ in $\mathbb{U}_{d}$. Then, for $|z| \leq r_{4}$,

$$
\left|f^{\prime}(z)\right| \leqq\left|g^{\prime}(z)\right|
$$

where $r_{4}$ is the positive smallest root of the equation

$$
\left(1-2 r^{2}-5 r\right)(1-\sinh r)-2 r \cosh r=0 .
$$

Proof. Let $g(z) \in \mathscr{F}_{\mathcal{S} \mathscr{L}}$. Then, a holomorphic function $w(z)$ in $\mathbb{U}_{d}$ occurs with $w(0)=0$ and $|w(z)| \leq|z|$ so that

$$
\frac{g(z)}{z q(z)}=\sqrt{1+w(z)}
$$

Now, after simple calculations, we have

$$
\frac{z g^{\prime}(z)}{g(z)}=1+\frac{z q^{\prime}(z)}{q(z)}+\frac{z w^{\prime}(z)}{2(1+w(z))}
$$


Using (23), we obtain

$$
\begin{aligned}
\frac{|z|\left|w^{\prime}(z)\right|}{2(1-|w(z)|)} & \leq \frac{|z|(1+|w(z)|)}{2\left(1-|z|^{2}\right)} \leq \frac{|z|(1+|z|)}{2\left(1-|z|^{2}\right)} \\
& =\frac{|z|}{2(1-|z|)} \leq \frac{r}{2(1-r)} .
\end{aligned}
$$

By virtue of (23) and Lemma 1, we obtain

$$
\begin{aligned}
\left|\frac{g(z)}{g^{\prime}(z)}\right| & \leqq \frac{|z|}{1-\left|z p^{\prime}(z) / p(z)\right|-\left|z w^{\prime}(z) / 2(1+w(z))\right|} \\
& \leqq \frac{2 r\left(1-r^{2}\right)(1-\sinh r)}{2\left(1-r^{2}\right)(1-\sinh r)-2 r \cosh r-r(1+r)(1-\sinh r)} .
\end{aligned}
$$

Now, using (31) and (53) in (30), we get

$$
\left|f^{\prime}(z)\right| \leqq\left[\varphi(z)+\frac{2 r\left(1-|\varphi(z)|^{2}\right)(1-\sinh r)}{2\left(1-r^{2}\right)(1-\sinh r)-2 r \cosh r-r(1+r)(1-\sinh r)}\right]\left|g^{\prime}(z)\right| .
$$

The required result follows directly using similar calculations as Theorem 2 along with the use of (33).

\section{Conclusion}

For some particular subfamilies of holomorphic functions which are connected with different shapes of image domains, we studied the problems of majorization. These problems can be examined for some other families such as for the families of meromorphic functions as well as for the families of harmonic functions.

\section{Data Availability}

No data were used to support this study.

\section{Conflicts of Interest}

The authors declare no conflict of interest.

\section{Authors' Contributions}

All authors contributed equally to this research paper.

\section{Acknowledgments}

This project was funded by the Deanship of Scientific Research (DSR), King Abdulaziz University, under grant No. (DF-763-830-1441). Therefore, the authors gratefully acknowledge DSR technical and financial support.

\section{References}

[1] L. Bieberbach, "Über dié koeffizienten derjenigen Potenzreihen, welche eine schlichte Abbildung des Einheitskreises vermitteln," Sitzungsberichte Preussische Akademie der Wissenschaften, vol. 138, pp. 940-955, 1916.
[2] L. De-Branges, "A proof of the Bieberbach conjecture," Acta Mathematica, vol. 154, no. 1-2, pp. 137-152, 1985.

[3] M. S. Roberston, "Quasi-subordination and coefficient conjectures," Bulletin of the American Mathematical Society, vol. 76, no. 1, pp. 1-9, 1970.

[4] T. H. MacGregor, "Majorization by univalent functions," Duke Mathematical Journal, vol. 34, no. 1, pp. 95-102, 1967.

[5] O. Altintas and H. M. Srivastava, "Some majorization problems associated with p-valently starlike and convex functions of complex order," East Asian Mathematical Journal, vol. 17, no. 2, pp. 175-183, 2001.

[6] N. E. Cho, Z. Oroujy, E. A. Adegani, and A. Ebadian, "Majorization and coefficient problems for a general class of starlike functions," Symmetry, vol. 12, no. 3, p. 476, 2020.

[7] P. Goswami and M. K. Aouf, "Majorization properties for certain classes of analytic functions using the Sălăgean operator," Applied Mathematics Letters, vol. 23, no. 11, pp. 1351-1354, 2010.

[8] S. P. Goyal and P. Goswami, "Majorization for certain classes of analytic functions defined by fractional derivatives," Applied Mathematics Letters, vol. 22, no. 12, pp. 1855-1858, 2009.

[9] S. P. Goyal and P. Goswami, "Majorization for certain classes of meromorphic functions defined by integral operator," Annales UMCS, Mathematica, vol. 66, no. 2, pp. 57-62, 2016.

[10] S.-H. Li, H. Tang, and E. Ao, "Majorization properties for certain new classes of analytic functions using the Salagean operator," Journal of Inequalities and Applications, vol. 2013, no. 1, Article ID 86, 2013.

[11] T. Panigraht and R. El-Ashwah, "Majorization for subclasses of multivalent meromorphic functions defined through iterations and combinations of the Liu-Srivastava operator and a meromorphic analogue of the Cho-Kwon-Srivastava operator," Filomat, vol. 31, no. 20, pp. 6357-6365, 2017.

[12] J. K. Prajapat and M. K. Aouf, "Majorization problem for certain class of p-valently analytic function defined by generalized fractional differintegral operator," Computers \& Mathematics with Applications, vol. 63, no. 1, pp. 42-47, 2012.

[13] H. Tang, M. K. Aouf, and G. Deng, "Majorization problems for certain subclasses of meromorphic multivalent functions associated with the Liu-Srivastava operator," Filomat, vol. 29, no. 4, pp. 763-772, 2015.

[14] H. Tang, H. M. Srivastava, S.-H. Li, and G.-T. Deng, "Majorization results for subclasses of starlike functions based on the sine and cosine functions," Bulletin of the Iranian Mathematical Society, vol. 46, no. 2, pp. 381-388, 2020.

[15] W. C. Ma and D. Minda, "A unified treatment of some special classes of univalent functions," Proceedings of the Conference on Complex Analysis, Z. Li, F. Ren, L. Yang, and S. Zhang, Eds., , pp. 157-169, Int. Press, 1994.

[16] W. Janowski, "Extremal problems for a family of functions with positive real part and for some related families," Annales Polonici Mathematici, vol. 23, no. 2, pp. 159-177, 1970.

[17] M. Arif, K. Ahmad, J.-L. Liu, and J. Sokół, “A new class of analytic functions associated with Sălăgean operator," Journal of Function Spaces, vol. 2019, Article ID 6157394, 8 pages, 2019.

[18] K. I. Noor and M. Arif, "Mapping properties of an integral operator," Applied Mathematics Letters, vol. 25, no. 11, pp. 1826-1829, 2012.

[19] J. Sokól and J. Stankiewicz, "Radius of convexity of some subclasses of strongly starlike functions," Zeszyty Naukowe Politechniki Rzeszowskiej, vol. 19, pp. 101-105, 1996. 
[20] N. E. Cho, V. Kumar, S. S. Kumar, and V. Ravichandran, "Radius problems for starlike functions associated with the sine function," Bulletin of the Iranian Mathematical Society, vol. 45, no. 1, pp. 213-232, 2019.

[21] R. Mendiratta, S. Nagpal, and V. Ravichandran, "On a subclass of strongly starlike functions associated with exponential function," Bulletin of the Malaysian Mathematical Sciences Society, vol. 38, no. 1, pp. 365-386, 2015.

[22] L. Shi, H. M. Srivastava, M. Arif, S. Hussain, and H. Khan, “An investigation of the third Hankel determinant problem for certain subfamilies of univalent functions involving the exponential function," Symmetry, vol. 11, article 598, 2019.

[23] S. Kumar and V. Ravichandran, "A subclass of starlike functions associated with a rational function," Southeast Asian Bulletin of Mathematics, vol. 40, no. 2, pp. 199-212, 2016.

[24] K. Bano and M. Raza, "Starlike functions associated with cosine functions," Bulletin of Iranian Mathematical Society, 2020.

[25] A. Abdullah, M. Arif, M. A. Alghamdi, and S. Hussain, "Starlikness associated with cosine hyperbolic function," Mathematics, vol. 8, no. 7, article 1118, 2020. 